\title{
20 Innere Verhältnisse im Chanat der Krim
}

\begin{abstract}
Nur wenige der edleren oder einfacheren Tataren kümmern sich um die Vermögensverhältnisse, auch wenn sie fruchtbaren Boden haben. Die meisten bebauen weder Äcker noch pflanzen sie etwas an. An Pferden, Kamelen, Ochsen, Kühen, Kleinvieh und Schafen haben sie Überfluss und davon ernähren sie sich. Die edleren haben dennoch Brot und Fleisch zur Speise, als Trank aber Branntwein und Honigwein. [...] Einige aber sind dem Khan oder den höhergestellten Tataren, damit sie nur Nahrung haben und sich kleiden können, wie Mietlinge dienstbar - nur ohne Lohn. Die übrigen aber, und das sind die meisten, sind stets müßig. ${ }^{1}$
\end{abstract}

Auch diese Passage aus der Krim-Beschreibung des polnischen Gesandten Broniewski aus dem Jahr 1579 transportiert ein Bild der ,fremden` krimtatarischen Bevölkerung von überzeitlicher Gültigkeit: Aus der Perspektive eines christlichen Europäers werden die allermeisten Krimtataren (wieder nur Männer) unabhängig ihres sozialen Standes als wenig umtriebig, ja sogar faul beschrieben. Obgleich es die Natur offenbar gut mit der Krim gemeint hat, denn es gibt fruchtbare Äcker und eine reichhaltige Fauna, nutzten die BewohnerInnen dieses Kapital nicht, so der mehr oder weniger implizite Vorwurf. Nur wenige gingen aus purer Not einer mit Naturalien entlohnten Arbeit (als dienstbare „Mietlinge“) nach, die meisten aber seien „stets müßig.“ In seiner Krim-Beschreibung verwickelte sich der Autor übrigens durchaus in Widersprüche, berichtete er doch an anderer Stelle über „[v]orzügliche Obstgärten, Weingärten, Gemüsegärten“, die es „ohne Zahl“ gebe ${ }^{2}$ - und für die auch irgendjemand hat Sorge tragen müssen, wachsen Obst, Gemüse und Weinreben doch nicht ohne aufmerksame Pflege. Broniewski oder der bereits oben zitierte Reiseschriftsteller de Windt, der die Krim mehr als dreihundert Jahre später besuchen sollte und die Krimtataren der Südküste wegen des dortigen angenehmen Klimas ebenfalls nicht für gerade fleißig hielt - sie stehen in einer Reihe mit vielen hier ungenannten Autorinnen und Autoren aus dem sog. christlichen Abendland. Broniewskis Aussage über ,faule 'Muslime transportiert ein bekanntes Stereotyp über ,den Orientalen' an sich, ${ }^{3}$ dass in dem Zitat allein der Hinweis auf den Alkoholkonsum der Muslime in Form von Brannt- und Honigwein erstaunt; wobei, wie schon erwähnt, die muslimische Krimbevölkerung ohnehin nicht nur Alkohol produzierte, sondern diesen auch trank. Auch hoch-

1 Broniovius (2011), 105.

2 Broniovius (2011), 79.

3 Darauf hat bekanntlich am Beispiel der professionalisierten Orientalistik besonders nachdrücklich Edward Said hingewiesen, u. a. Said (1978). Zur Rolle des „Orientalismus“ in der Osteuropäischen Geschichte vgl. u.a. Jobst (2000); Schimmelpenninck van der Oye (2010).

Ә OpenAccess. (C) 2020 Kerstin S. Jobst, publiziert von De Gruyter. (cc))BY Dieses Werk ist lizenziert unter der Creative Commons Attribution 4.0 International. https://doi.org/10.1515/9783110520620-022 
gradig gebildete, sich der objektiven Wissenschaft verpflichtet fühlende Reisende wie Peter Simon Pallas (1741-1811), ein in Preußen geborener Forscher in russischen Diensten und Mitglied der dortigen Akademie der Wissenschaften, bedauerte zutiefst, dass Krimtataren mit dem natürlichen Reichtum der Halbinsel angeblich nichts anzufangen wüssten. ${ }^{4}$ Als er in den Jahren 1793 und 1794 diese im Auftrag der Zarin Katharina II. besuchte, um die dortige ökonomische Lage zu prüfen und in der Folge St. Petersburg Vorschläge zur künftigen Politik zu unterbreiten, ${ }^{5}$ klagte er:

Es ist zu bedauern, daß alle die schönen und warmen Thäler des südlichen Ufers, theils mit unnützen, unthätigen, ja wohl gar in gewissen Fällen gefährlichen Tataren besetzt sind, die mehr zu verwüsten als anzupflanzen wissen, theils, was der Krone zugehörte, an solche Eigenthümer verschenkt worden ist, welche weder das Vermögen, noch den guten Willen haben, in so günstigen Gegenden Anlage für das gemeine Beste zu machen. ${ }^{6}$

Aus ökonomischen und sicherheitsstrategischen Überlegungen empfahl er der russischen Administration die Enteignung tatarischer Landbesitzer und ihre Deportation ins Landesinnere, denn sie seien ,unnütze und unwürdige BewohnerInnen in paradisischen Thälern, in welchen sie sonst immer die ersten und fertigsten Aufrührer gegen Russland waren.“ An ihrer Stelle seien ,industriöse Colonien“ mit Neusiedlern anzulegen. ${ }^{7}$ Dies war eine Politik, der die russischen Machthaber in den nächsten Jahrzehnten übrigens folgten (vgl. Kapitel 25). Die krimtatarische Bevölkerung wurde aus westlicher Perspektive recht übereinstimmend also als wenig nützlich und - hier war der Bezug auf die bereits geschilderte, historisch gewachsene Überzeugung, ein jeder Krimtatare sei zugleich ein Krieger - als gefährlich bezeichnet. Die im europäischen Diskurs seit der Frühen Neuzeit tief verankerte Vorstellung, beim Krim-Chanat handele es sich um einen Parasitenstaat, der allein auf Raub, Beutemachen und Sklavenhandel fuße, erwies sich demnach als sehr wirkungsmächtig. Dessen innere Verfasstheit, sein Bevölkerungsgemisch und auch die wirtschaftlichen und militärischen Strukturen waren aber ungleich komplexer, als dieses Bild glauben macht.

\footnotetext{
4 Peter Simon Pallas, Bemerkungen auf einer Reise in die südlichen Statthalterschaften des Russischen Reiches in den Jahren 1793 und 1794, im Folgenden zitiert nach der Ausgabe Pallas (1967). Erstmalig 1799 auf Deutsch erschienen, wurde dieses Werk in der Folge in fast alle europäischen Sprachen - auch ins Russische - übersetzt. Zur Person vgl. Wendland (1992). Pallas bereiste nicht nur die Krim, sondern unternahm auch umfangreiche Expeditionen in andere Teile des Russländischen Reiches, u.a. in den Ural und nach Sibirien.

5 Vgl. dazu Jobst (2007b), 117-122.

6 Pallas (1967), Bd. 2, 259.

7 Pallas (1967), Bd. 2, $349 \mathrm{f}$.
} 
Dass vielfältige Akkulturationsprozesse auf der Krim zu allen Zeiten an der Tagesordnung gewesen sind und dort eine besondere Melange von Kulturen und Religionen entstehen ließen, wurde in diesem Buch bereits wiederholt thematisiert und ist geradezu ein roter Faden in der Geschichte der Halbinsel. Die Zeit des Krim-Chanats stellt keine Ausnahme dar, so dass die in wissenschaftlichen Debatten seit einigen Jahren so lebhaft diskutierten sog. hybriden Kulturen sich eben auch besonders gut am Beispiel der Krim in der Frühen Neuzeit beobachten und erforschen lassen. ${ }^{8}$ Lange Zeit überwiegend nomadisch wirtschaftende Gruppen wie die Nogaier wurden zum Teil sesshaft, andere ethnische und religiöse Gruppen - Nachfahren der italienischen Kolonisten, Armenier, Juden, Karäer, Griechen etc. - tatarisierten sich partiell, etwa in Sprache und Kleidung. Auch veränderten sich die religiösen Verhältnisse: Besonders im 16. Jahrhundert kam es in den Küstengebieten, Städten und Bergregionen vermehrt zu Konversionen bislang nicht-muslimischer Einwohner zum Islam. Und im Unterschied zur Zeit des Russländischen Imperiums, als exogenes Heiratsverhalten (also Eheschließungen zwischen Angehörigen unterschiedlicher Religionen) eine absolute Ausnahme darstellte9, war dieses Phänomen in der Frühen Neuzeit häufiger zu beobachten. Der Glaubenswechsel brachte den bisherigen NichtmuslimInnen bekanntlich wirtschaftliche Vorteile, entfielen doch die im Osmanischen Reich wie auch im Chanat erhobenen Sondersteuern. Zuweilen wurde für die muslimische Seite die große Zahl der Übertritte sogar zum Problem, da dies mit weniger Steuereinnahmen einherging. ${ }^{10}$ In jedem Fall ist festzuhalten, dass bis wenige Jahre vor der russischen Annexion im Chanat nicht-muslimische Bevölkerungsgruppen in aller Regel mit ihren muslimischen Nachbarn in friedlicher Koexistenz lebten; zu interreligiösen Konflikten größeren Ausmaßes kam es erst später.

In der Geschichte der Halbinsel sind Vielfältigkeit und eine daraus erwachsende bemerkenswerte kulturelle Gemengelage angelegt, die aber zugleich die Entwicklung protonationaler Kulturen nicht verhinderte. Und dies galt eben auch für die spezifische, krimtatarische Kultur, von der sich selbst russische AutorInnen des 19. Jahrhunderts ungeachtet ihrer Vorbehalte gegenüber allem ,Tatarischen“ beeindruckt zeigten. ${ }^{11}$ Trotz aller über die Zeit - und am entschlossensten in den

8 Vgl. dazu den Reader kanonischer Texte zur Debatte: Bronfen (1997).

9 Nach Williams (2001), 124, gibt es keinerlei Erkenntnisse über christlich-tatarische Heiraten in zarischer Zeit; er interpretiert dies als einen Akt der Verteidigung muslimischer Identität seitens der krimtatarischen Bevölkerung. Vgl. demgegenüber Jobst (2007b), 212-215, die einige Fälle solcher Verbindungen auf der Grundlage russischer Quellen dokumentiert hat.

10 So zumindest die Interpretation von Fisher (1981), 141, zur Situation in der zum Osmanischen Reich gehörenden Stadt Kefe.

11 Jobst (2007b), 192-195. 
Jahren nach dem Zweiten Weltkrieg im Spätstalinismus - erfolgten Zerstörungen bedeutender Moscheen, Archive oder Teile des heute sehr viel kleineren Palasts von Bağçasaray sind die Überreste krimtatarischen kulturellen Schaffens immer noch beeindruckend. Das Herzstück war und ist der Chanspalast, mit dessen Bau der Chan Sahib I Giray (1501-1551) begonnen hatte. ${ }^{12}$ Wenige Jahrzehnte nach Baubeginn, im ausgehenden 16. Jahrhundert, kam Broniewski nicht umhin, die Palastanlage und die Ortschaft zu loben: Die Stadt an sich sei nicht unbedeutend, das Gebäude selbst aus „,vorzüglichen Steinen“ gefertigt und „auf verschwenderische und großartige Weise mit Gebäuden, Tempeln, Grabmälern und Bädern ausgeschmückt."13 Auch Reisende aus dem Osmanischen Reich, die mit der prächtigen Architektur des Topkapı-Palastes in Istanbul vertraut waren, an dem sich die persischen, osmanischen und italienischen Architekten auf der Krim im Übrigen orientiert hatten, zeigten sich beeindruckt: Evliyâ Çelebi, der ein gutes dreiviertel Jahrhundert nach Broniewski die Krim bereiste, lobte ebenfalls die gelungene Architektur des Palastes und zollte der Infrastruktur, den Dörfern und Städten auf der Krim Anerkennung. ${ }^{14}$

Offenbar ließ sich die elaborierte krimtatarische Kultur selbst von kritischen, dem Chanat eher ablehnend gegenüberstehenden Autoren nicht überzeugend unter dem Begriff „barbarisch“ subsumieren; zu beeindruckend war vieles, was sie auf der Halbinsel sehen konnten. Leichter fiel es zumindest christlichen Autoren aufgrund ihrer spezifischen (Kriegs-)Erfahrungen mit dem Krim-Chanat, den Ursprung dieses Wohlstands auf Beutezüge und Tributeintreibungen zu reduzieren. Selbst der profunde Kenner der krimtatarischen Geschichte Alan Fisher bemerkte an einer Stelle seines wichtigen Buches, dass die Wirtschaft des Chanats zuvorderst darauf sowie auf den Unterstützungszahlungen der Hohen Pforte beruht habe. ${ }^{15}$ Auch wenn ohne Zweifel diese Einkünfte von großer Relevanz waren, so sollte die agrarische Leistungsfähigkeit auch der muslimischen KrimbewohnerInnen nicht unterschätzt werden: Wie bereits dargestellt, waren die BewohnerInnen der nördlichen Steppen in der Vieh- und zum Teil auch in der Getreidewirtschaft tätig (s.o.). Gerade in der Bergregion war neben der Imkerei die

12 Zur Geschichte und Architektur des vermutlich um 1540 begonnenen Palasts, der in mehreren Etappen erbaut wurde, vgl. neben Fisher (1978), 29f. die offizielle Website Bachčisarajski zapovednik (2018). Interessant ist auch die russischsprachige Wikipedia-Seite: Der Palast wird nicht, wie im russischen und sowjetischen Diskurs lange dominierend, als Bauwerk minderer Güte eingeschätzt, sondern im Gegenteil: Er sei ein Denkmal von universeller Bedeutung. Vgl. Chanskij dvorec (2018).

13 Broniovius (2011), 79.

14 Vgl. Hillebrand (2017), 53.

15 Fisher (1978), 16. 


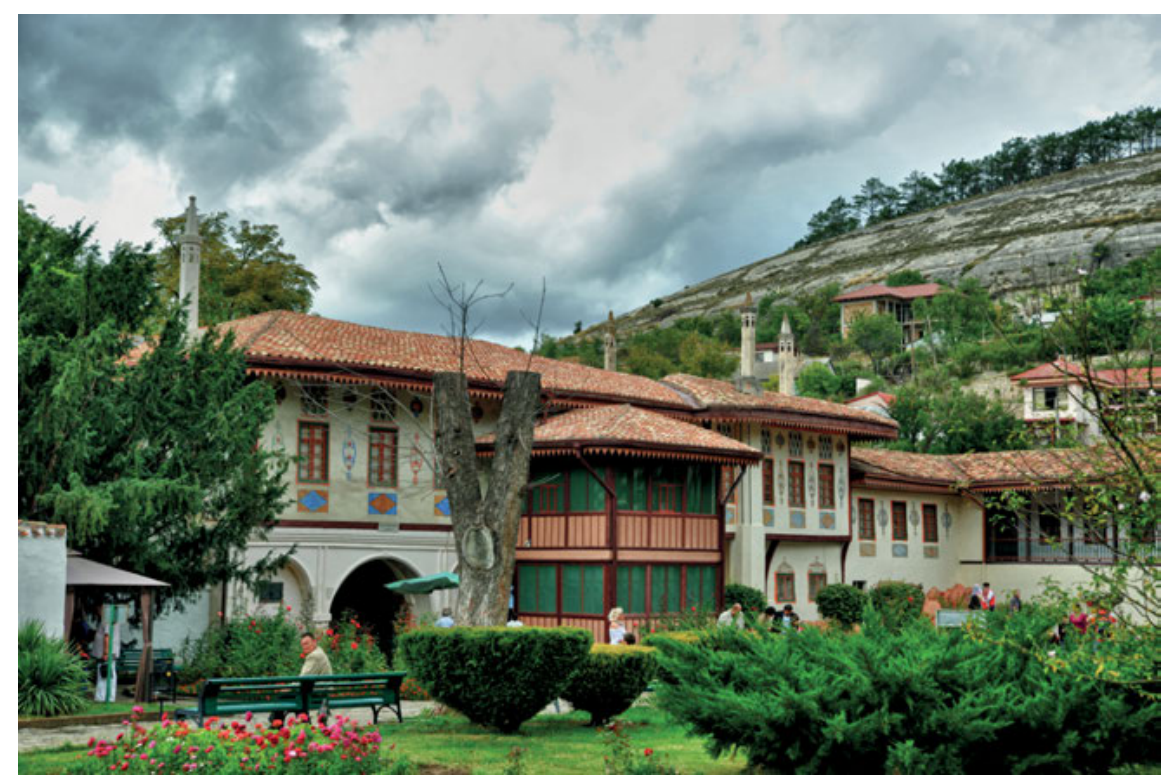

Abb. 6: Chan-Palast von Bağçasaray

Gartenwirtschaft, die ja auch Broniewski bemerkt hatte, ein wichtiger Faktor. ${ }^{16}$ Der große sowjetische Archäologe und Kunsthistoriker des nördlichen Schwarzmeerraumes Anatolij L. Jakobson (1906-1984) bescheinigte den sesshaften Tataren Erfolge auf dem Gebiet des Obst- und Weinanbaus, des Handwerks und der Architektur. Er führte ihre Fähigkeiten allerdings darauf zurück, dass sie sich schon früh an die Nachfahren der griechischen Kolonisten assimiliert - also quasi von diesen gelernt - hätten. ${ }^{17}$

Ohne Zweifel kam dem Militärwesen im Chanat eine zentrale Bedeutung zu. So war jeder männliche Tatare seit dem 16. Jahrhundert verpflichtet, dem Ruf des Krim-Chans zu folgen, wenn dieser den Wunsch äußerte, ,sich aufs Pferd zu setzen“, d.h. einen Kriegszug zu unternehmen. ${ }^{18}$ Bei Zuwiderhandlung, Nichterscheinen am Sammelplatz oder Feigheit vor dem Feind drohten, wie in der Zeit nicht nur bei MuslimInnen üblich, Strafen bis zum Tod. ${ }^{19}$ Ein stehendes Heer unterhielt das Chanat also nicht - und war dabei in guter Gesellschaft, bildeten

16 Vgl. zur landwirtschaftlichen Produktion auf der Krim während des Chanats Vozgrin (1992), 156-158.

17 Jakobson (1973), 148.

18 Chartachaj (1866/1867), 1, 207.

19 Collins (1975), 259. 
sich solche in den aufkommenden Territorialstaaten der Frühen Neuzeit doch erst allmählich heraus. Stehende Heere waren eine Reaktion auf häufig unzuverlässige Söldner, zu denen letztlich auch die bereits beschriebenen Registerkosaken in Polen-Litauen zählten. Üblich waren zudem Milizarmeen. ${ }^{20}$ Die sehr schlagkräftige und gefürchtete Armee der Krimtataren bestand hauptsächlich aus Milizen, denn nur eine vergleichsweise kleine Zahl betrieb ausschließlich das Kriegshandwerk - sie war damit im Kontext der Zeit eher eine Armee älteren Typs. Doch eine effektive Mobilisierungspolitik machte das Zusammenziehen zehntausender Krieger - in Einzelfällen vermutlich bis zu 80.000 - binnen sehr kurzer Zeit möglich, was ein großer Vorteil gegenüber gegnerischen Armeen war. ${ }^{21}$

Die Mehrheit der krimtatarischen Männer widmete sich also entgegen den landläufigen Meinungen christlicher Autoren nicht ausschließlich dem Kriegsdienst. Nur ein kleiner Prozentsatz der Krimtataren konnte es sich nämlich leisten, ihr Leben ganz dem Militär zu widmen; die anderen benötigten zusätzliche Einkommensquellen. Durch den bereits in der Kindheit engen Kontakt mit Pferden zumindest bei den (halb-)nomadischen Tataren und dem ebenfalls früh geübten Gebrauch ihrer wichtigen Waffe, dem Reflexbogen, brachten es die Armeen des Chans dennoch zu hoher Perfektion. ${ }^{22}$ Wenn die Chane aber nicht zum Kriegszug riefen, hüteten männliche Krimtataren zumeist ihr Vieh, bewirtschafteten ihr Land oder arbeiteten als Handwerker.

Grund und Boden wurden übrigens gemeinschaftlich bearbeitet, und gemeinsam wurden auch die Steuern an den jeweiligen Grundbesitzer, der zumeist einem der einflussreichen Clans angehörte, entrichtet. ${ }^{23}$ Ein entscheidender Unterschied zu den angrenzenden Gebieten Polen-Litauens und des Moskauer Reiches war, dass die krimtatarischen Bauern keine Leibeigenen waren, also persönlich frei und räumlich zumindest de jure mobil; daneben gab es aber die ja schon hinlänglich beschriebenen Sklaven, die als ,sprechender Besitz' galten. Auch nach 1783 und der Annexion der Krim durch das Russländische Reich wurde die krimtatarische Bevölkerung übrigens nicht in die Leibeigenschaft gezwungen. Neben Bağçasaray und dem zum Osmanischen Reich gehörenden Kefe gab es, wie bereits in vortatarischer Zeit, ein differenziertes urbanes Leben, an dem die tatarische Bevölkerung großen Anteil hatte, vielfach sogar die Mehrheit stellte; zu den wichtigen Städten gehörten u.a. das im Westen der Halbinsel gelegene Gözleve (türk.; krimtat.: Kezlev; das heutige Jevpatorija/ Evpatorija [ukr./russ.]), das

20 Dazu im Überblick Papke (1983).

21 Collins (1975), $259 \mathrm{f}$.

22 Collins (1975), 262-264.

23 Magocsi (2014), 43. 
für das Chanat ein wichtiger Handelsplatz wurde, das im Landesinnere gelegene Aqmescit (krimtat.; das heutige Simferopol' [ukr./russ.]), oder Qarasuvbazar (krimtat.; heute: Bilohirs'k/Belogorsk [ukr./russ.]). Die vielfach sprachlich und habituell tatarisierten Armenier, Georgier, Griechen oder Karäer (letztere primär in Qarasuvbazar) hatten großen Einfluss auf Handel und Finanzgeschäfte. ${ }^{24}$ Vor allen Dingen in den Küstenstädten waren übrigens nicht alle tatarischen BewohnerInnen Subjekte des Krim-Chans, sondern häufig UntertanInnen des Sultans. Im Verlauf der Frühen Neuzeit stellte die krimtatarische Bevölkerung insgesamt - also die der Küsten- und Gebirgsregion, die von Ethnologen zumeist als „Tat“ bzw. „Yalyboyu“ bezeichnet werden²5, sowie die Nogaier im Norden - die Mehrheit der Bevölkerung auf der Krim. Magocsi schätzt, dass auf dem Territorium des Krim-Chanats insgesamt, welches ja weitaus größer war als die Halbinsel, in der Mitte des 16. Jahrhunderts eine halbe Million Menschen lebte. ${ }^{26}$

Auf die Begrenzung der Macht der Chane nicht nur durch den osmanischen Sultan, sondern auch durch die Clans wurde bereits hingewiesen. Die hervorgehobene Position der Familien war in den Nachfolgestaaten der Goldenen Horde eine übliche Erscheinung, im Osmanischen Reich hingegen unbekannt. Die ClanHäupter waren Teil des sog. Divan, des Beratergremiums des Chans. Neben diesem Divan existierte der Kurultay, die Clan-Versammlung aus Großgrundbesitzern und Kriegsherren, der u.a. als Sprachrohr gegenüber dem Sultan fungierte und überragenden Einfluss auf die Staatsgeschäfte ausübte. ${ }^{27}$ Bis zum Ende des Chanats scheiterten alle Giray-Chane daran, den Einfluss der Sippen zu beschneiden und ihre eigene Zentralgewalt zu stärken.

Es steht zu fragen, wie sich das Krim-Chanat in der Struktur der frühneuzeitlichen europäischen Staaten ausnahm, wo sich zumindest im mitteleuropäischen Kontext in den Städten differenzierte Rechts- und Organisationsformen entwickelten und sich andernorts der Territorialstaat mit einem Souverän an der Spitze allmählich herausbildete. Diese Tendenzen sind im Krim-Chanat nicht feststellbar. Auch eine Einordnung in den ost(mittel)europäischen Kontext der

24 Fisher (1978), 30.

25 Zur Ethnogenese der Krimtataren vgl. Williams (2001), 7-39; Vojtovyč (2009). Die Unterscheidung zwischen „Tat“ und „Yaliboyu“ erfolgt entlang linguistischer Grenzen, da letztere einen oghusischen, also südwesttürkischen Dialekt sprechen.

26 Magocsi (2014), 43. Demnach hätte sich die Bevölkerungszahl bis in das 18. Jahrhundert nicht erhöht; vgl. hierzu die Gegenüberstellung der verschiedenen Schätzungen bei Williams (2001), $69 \mathrm{f}$.

27 Davies B. (2007), 23, ist der Auffassung, dass die zahlreichen nach Norden unternommenen Sklavenzüge letztlich auch ein Resultat der übergroßen Macht der Clans im Khanat gewesen seien: „[T]he Chan had to offer them frequent opportunities to raid for prisoners for ransom“, andernfalls hätten diese auf eigene Faust gehandelt. 
Zeit gelingt nicht wirklich, so dass von einer Sonderstellung des Chanats auszugehen ist, die Alan Fisher folgendermaßen umreißt: „Unlike its neighbors (i.e. Poland-Lithuania, Moscow, and the Ottoman Empire) the Khanate was not a feudal monarchy, an absolute monarchy, a patrimonial state, or an oriental despotism. It was something quite different, perhaps without European or eastern European parallel." ${ }^{\text {28 }}$ Das Chanat entzieht sich also auf diesem Feld einer eindeutigen Bewertung, zumal das bereits ausführlich dargelegte komplexe Verhältnis zum Osmanischen Reich nicht außer Acht gelassen werden darf. In jedem Fall war das Chanat nicht der barbarische, despotische Parasitenstaat, als der er oft beschrieben wurde.

28 Fisher (1978), 21. 